\title{
THE $H^{p}$ CLASSES FOR $\alpha$-CONVEX FUNCTIONS
}

\author{
P. J. EENIGENBURG ${ }^{1}$ AND S. S. MILLER
}

Abstract. Given $\alpha>0$, we determine the $H^{p}$ class to which an $\alpha$-convex function belongs.

Introduction. In this paper we continue the study ([4], [5], [6], [7]) of $\mathscr{M}_{\alpha}$, the class of $\alpha$-convex functions. Our purpose is to obtain the $H^{p}$ class to which a given $\alpha$-convex function belongs.

Definition. Let $f(z)=z+\sum_{n=2}^{\infty} a_{n} z^{n}$ be analytic in the unit disc $D$, with $(f(z) / z) f^{\prime}(z) \neq 0$ there, and let $\alpha$ be a real number. Then $f(z)$ is said to be $\alpha$-convex in $D$ if and only if

$$
\operatorname{Re}\left[(1-\alpha) \frac{z f^{\prime}(z)}{f(z)}+\alpha\left(1+\frac{z f^{\prime \prime}(z)}{f^{\prime}(z)}\right)\right]>0 .
$$

It is known [5] that if $f(z)$ is $\alpha$-convex then $f(z)$ is starlike and univalent in $D$. Moreover, if $\alpha \geqq 1$ then $f(z)$ is convex in $D$. In our investigation we shall employ the $H^{p}$ results concerning starlike and convex functions [3]. We shall also make use of the following subclasses, first introduced by Reade [11], of the class of close-to-convex functions.

Definition. Let $K_{\beta}$ denote the class of functions $f(z)$, analytic in $D$, for which there exists a convex function $h(z)$ such that

$$
\left|\arg \left(f^{\prime}(z) / h^{\prime}(z)\right)\right| \leqq \beta \pi / 2 .
$$

The following theorem can be found in [7].

THEOREM 1. If $f(z) \in \mathscr{M}_{\alpha}(0 \leqq \alpha \leqq 1)$, then $f(z) \in K_{1-\alpha}$.

Proof. Since $f(z) \in \mathscr{M}_{\alpha}$ one easily checks that $f(z)\left(z f^{\prime}(z) / f(z)\right)^{\alpha}$ is starlike, so that

$$
h(z) \equiv \int_{0}^{z} \frac{f(w)}{w}\left(\frac{w f^{\prime}(w)}{f(w)}\right)^{\alpha} d w
$$

Presented to the Society, January 26, 1973; received by the editors July 31, 1972 and, in revised form, August 31, 1972.

AMS (MOS) subject classifications (1970). Primary 30A32; Secondary 30A72.

${ }^{1}$ The first author acknowledges support received under a Western Michigan University Faculty Research Fellowship.

(C) American Mathematical Society 1973 
is convex. The result then follows from the identity

$$
f^{\prime}(z) / h^{\prime}(z)=\left(z f^{\prime}(z) / f(z)\right)^{1-\alpha}
$$

and the fact that $f(z)$ is starlike.

We now make the following two observations.

(a) If $f(z) \in \mathscr{M}_{\alpha}(\alpha>0)$, then $f(z)$ is continuous in $\bar{D}$ (if $\infty$ is allowed as a value) and assumes no finite value more than once.

(b) If $f(z) \in \mathscr{M}_{\alpha}(\alpha>0)$, and $L(r)$ denotes the length of $\{w=f(z):|z|=r\}$, then $L(r)=O(M(r))$ as $r \rightarrow 1$.

Pommerenke [10] obtained the results in (a) and (b) for $f(z) \in K_{\beta}$, $\beta<1$, and consequently by Theorem 1 they follow for $f(z) \in \mathscr{M}_{\alpha}, 0<\alpha<1$. In case $\alpha \geqq 1, f(z)$ is convex and the results (a) and (b) are well known.

We further observe from (a) that none of the classes of starlike functions of a positive order (less than unity) is contained in $\mathscr{M}_{\alpha}$ for some positive $\alpha$. This follows from the existence of bounded functions, starlike of any given order (less than unity), which do not extend continuously to $\bar{D}$ [2]. The question of the order of starlikeness for the class $\mathscr{M}_{\alpha}$ remains open.

THEOREM 2. If $f(z) \in K_{\beta}$ and there exists a convex function $h(z)$, not of the form $h(z)=a+b z\left(1+z e^{i \tau}\right)^{-1}$, such that $\left|\arg \left(f^{\prime}(z) / h^{\prime}(z)\right)\right| \leqq \beta \pi / 2$, then there exists $\varepsilon=\varepsilon(f)>0$ such that

$$
f(z) \in H^{1 /(1+\beta)+\varepsilon} \quad \text { and } f^{\prime}(z) \in H^{1 /(2+\beta)+\varepsilon} .
$$

Proof. We first observe that if $\beta=0$ or $\beta=1$, these results are known [3]. Writing $f^{\prime}(z)=h^{\prime}(z) P(z)$ where $|\arg P(z)| \leqq \beta \pi / 2$, it follows that $P(z) \in H^{\lambda}, \forall \lambda, \lambda<1 / \beta$. Also, from Theorem 3 of [3], $h^{\prime}(z) \in H^{1 / 2+\delta}$ for some $\delta=\delta(h)>0$. Application of Hölder's inequality with

yields

$$
\begin{aligned}
& p=\left(\frac{1}{2}+\delta\right)(\beta+2-\delta), \\
& q=\left(\frac{1}{2}+\delta\right)(\beta+2-\delta)\left(\beta / 2+\delta \beta+3 \delta / 2-\delta^{2}\right)^{-1}
\end{aligned}
$$

$$
\int_{-\pi}^{\pi}\left|f^{\prime}(z)\right|^{(\beta+2-\delta)^{-1}} d \theta \leqq\left(\int_{-\pi}^{\pi}\left|h^{\prime}(z)\right|^{p /(\beta+2-\delta)} d \theta\right)^{1 / p}\left(\int_{-\pi}^{\pi}|P(z)|^{\alpha /(\beta+2-\delta)} d \theta\right)^{1 / \alpha} .
$$

If $\delta$ is sufficiently small, each of the integrals on the right remains bounded as $r$ tends to 1 . Hence there exists $\varepsilon=\varepsilon(f)>0$ such that $f^{\prime}(z) \in H^{1 /(\beta+2)+\varepsilon}$. By a well-known theorem of Hardy-Littlewood [1, p. 88], $f(z) \in H^{1 /(\beta+1)+\varepsilon}$ for a possibly different value of $\varepsilon$.

We require the following integral representation [6] for functions in $\mathscr{M}_{\alpha}, \alpha>0$ : the function $f(z)$ is in $\mathscr{M}_{\alpha}, \alpha>0$, if and only if there exists a 
starlike function $s(z)$ such that

$$
f(z)=\left[\frac{1}{\alpha} \int_{0}^{z}[s(\zeta)]^{1 / \alpha} \zeta^{-1} d \zeta\right]^{\alpha} .
$$

If $\alpha>2, f(z)$ is bounded [4] and $f^{\prime}(z) \in H^{1}$ (since $f(z)$ is convex).

Let us denote by $f_{\alpha}(z)$, the function obtained in (4) by letting $s(z)$ be the Koebe function, $k(z)=z(1-z)^{-2}$. It follows from (3) that $h(z)$ is of the form $z\left(1-z e^{i \tau}\right)^{-1}$ if and only if $f(z)=e^{-i \tau} f_{\alpha}\left(z e^{i \tau}\right)$. Theorems 1 and 2 then yield the following.

THEOREM 3. If $f(z) \in \mathscr{M}_{\alpha}(0 \leqq \alpha \leqq 1)$ and is not a rotation of $f_{\alpha}(z)$, then there exists $\varepsilon=\varepsilon(f)>0$ such that

$$
f(z) \in H^{1 /(2-\alpha)+\varepsilon} \text { and } f^{\prime}(z) \in H^{1 /(3-\alpha)+\varepsilon} .
$$

We remark that for $0 \leqq \alpha \leqq 2, f_{\alpha}(z) \notin H^{1 /(2-\alpha)}\left(H^{\infty}\right.$ if $\left.\alpha=2\right)$, although $f_{\alpha}(z) \in H^{\lambda}, \forall \lambda, \lambda<1 /(2-\alpha)$.

We now wish to establish Theorem 3 for $1<\alpha \leqq 2$. In this case $f(z)$ is both convex and starlike. These geometric properties give rise to the usual Herglotz formulas, which in turn yield probability measures $\mu_{1}$ and $\mu_{2}$, respectively. We can suppose these measures to be normalized so that

$$
\frac{1}{2}\left[\mu_{i}(t+0)+\mu_{i}(t-0)\right]=\mu_{i}(t), \quad \int_{-\pi}^{\pi} \mu_{i}(t) d t=0 \quad(i=1,2) .
$$

The normalization determines $\mu_{1}$ and $\mu_{2}$ uniquely; we shall call them the convex and starlike measures associated with $f(z)$, respectively.

LEMMA 4. Let $f(z)$ be an unbounded convex function and let $\mu_{1}$ be the convex measure associated with $f(z)$. If the maximum jump of $\mu_{1}(t)$ is $\gamma$, then $f(z) \in H^{\lambda}, \forall \lambda, \lambda<1 /(2 \gamma-1)$, and $f^{\prime}(z) \in H^{\lambda}, \forall \lambda, \lambda<1 / 2 \gamma$.

Proof. We first observe that since $f(z)$ is unbounded, $\gamma \geqq \frac{1}{2}[8, \mathrm{pp}$. 67-72]. The proof of Lemma 4 then follows from [3, p. 345].

THEOREM 5. Let $f(z)$ be an unbounded convex function. If the maximum jump of $\mu_{1}(t)$ is $\gamma$ then the maximum jump in $\mu_{2}(t)$ is $\gamma-\frac{1}{2}$.

Proof. Since $f(z)$ is convex, either (a) $f(z)$ is continuous on $\bar{D}$ with the exception of one point, say $z_{0}$, and at this point, $f(z) \rightarrow \infty$ as $z \rightarrow z_{0}$ with $|z| \leqq 1,\left|z_{0}\right|=1$, or (b) $f(D)$ is a domain whose boundary is two parallel lines [8]. In case (b), the result of Theorem 5 is obvious; hence, we assume case (a). We also assume that the maximum jump, $\gamma$, in $\mu_{1}(t)$ occurs at $t=0$. It follows by the continuity remark in (a) that the maximum jump in $\mu_{2}(t)$, call it $\beta$, also occurs at $t=0$. 
First, suppose $\gamma-\frac{1}{2}<\beta$. It is well known (see e.g. [12]) that $f(D)$ contains a sector of vertex angle $2 \pi \beta$. Hence, for some constant $C$,

$$
C((1+z) /(1-z))^{2 \beta}<f(z), \quad z \in D .
$$

Thus, $\int_{-\pi}^{\pi}|f(z)|^{1 / 2 \beta} d \theta$ becomes unbounded as $r$ tends to 1 , contradicting Lemma 4 (since $2 \gamma-1<2 \beta$ ).

We now suppose $\beta<\gamma-\frac{1}{2}$. Choosing $\delta$ such that $\beta<\delta<\gamma-\frac{1}{2}$, a result of Pommerenke [9] gives

$$
M(r, f)=O\left((1-r)^{-2 \delta}\right)
$$

The Cauchy formula yields

$$
M\left(r, f^{\prime}\right)=O\left((1-r)^{-2 \delta-1}\right) .
$$

On the other hand, since $z f^{\prime}(z)$ is starlike

$$
\left|z f^{\prime}(z)\right| \geqq K|z| /|1-z|^{2 \gamma},
$$

where $K$ is a constant [9]. But this contradicts the fact that $2 \delta+1<2 \gamma$. The result follows.

THEOREM 6. If $f(z) \in \mathscr{M}_{\alpha}(0 \leqq \alpha<2)$ and is not a rotation of $f_{\alpha}(z)$, then there exists $\varepsilon=\varepsilon(f)>0$ such that

$$
f(z) \in H^{1 /(2-\alpha)+\varepsilon} \text { and } f^{\prime}(z) \in H^{1 /(3-\alpha)+\varepsilon} .
$$

If $f(z) \in \mathscr{M}_{2}$ is unbounded, then $f(z)$ is a rotation of $f_{2}(z)$.

Proof. By Theorem 3, we may assume $\alpha>1$. We also take $f(z)$ unbounded. Let $\mu_{1}$ and $\mu_{2}$ be as in Theorem 5. Since $f(z) \in \mathscr{M}_{\alpha}, \mu=$ $(1-\alpha) \mu_{2}+\alpha \mu_{1}$ is a measure on [ $\left.-\pi, \pi\right]$. If $\gamma$ denotes the maximum jump in $\mu_{1}$, then the maximum jump in $\mu_{2}$ occurs at the same point and equals $\gamma-\frac{1}{2}$. Thus, the maximum jump in $\mu$ is $\gamma+\frac{1}{2}(\alpha-1)$. The rotations of $f_{\alpha}(z)$ are given by equating this number with unity; i.e., by setting $\gamma=\frac{1}{2}(3-\alpha)$.

Thus, let us assume $\frac{1}{2} \leqq \gamma<\frac{1}{2}(3-\alpha)$. We observe that if $\alpha=2$ then $\gamma=\frac{1}{2}$, and consequently there is only one unbounded function in $\mathscr{M}_{2}$ (up to rotations). ${ }^{2}$

By Lemma $4, f^{\prime}(z) \in H^{\lambda}(\lambda<1 / 2 \gamma)$. Since $1 / 2 \gamma>1 /(3-\alpha)$ we may choose $\varepsilon=\varepsilon(f)>0$ so that $1 / 2 \gamma>1 /(3-\alpha)+\varepsilon$. Hence, $f^{\prime}(z) \in H^{1 /(3-\alpha)+\varepsilon}$, and, for perhaps a different $\varepsilon, f(z) \in H^{1 /(2-\alpha)+\varepsilon}$.

2 M. O. Reade has pointed out to the authors that this result is also obtained by considering $g(z)=\sqrt{ }\left(f\left(z^{2}\right)\right)$, which is odd and convex, whenever $f(z) \in \mathscr{K}_{2}$. 


\section{REFERENCES}

1. P. L. Duren, Theory of $H^{p}$ spaces, Pure and Appl. Math., vol. 38, Academic Press, New York, 1970. MR 42 \#3552.

2. P. J. Eenigenburg, Boundary behavior of starlike functions, Proc. Amer. Math. Soc. 33 (1972), 428-433.

3. P. J. Eenigenburg and F. R. Keogh, The Hardy class of some univalent functions and their derivatives, Michigan Math. J. 17 (1970), 335-346.

4. S. S. Miller, Distortion properties of alpha-starlike functions, Proc. Amer. Math. Soc. (to appear).

5. S. S. Miller, P. Mocanu and M. O. Reade, All $\alpha$-convex functions are univalent and starlike, Proc. Amer. Math. Soc. 37 (1973), 553-554.

6. P. Mocanu, Une propriété de convexité généraliseé dans la théorie de la représentation conforme, Mathematica (Cluj) 11 (34) (1969), 127-133. MR 42 \#7881.

7. P. Mocanu and M. O. Reade, On generalized convexity in conformal mappings, Rev. Roumaine Math. Pures Appl. 16 (1971), 1541-1544.

8. V. Paatero, Über die konforme Abbildungen von Gebieten deren Ränder von beschränkter Drehung sind, Ann. Acad. Sci. Fenn. A33 (1931), no. 9.

9. Ch. Pommerenke, On starlike and convex functions, J. London Math. Soc. 37 (1962), 209-224. MR 25 \#1279.

10. - On close-to-convex analytic functions, Trans. Amer. Math. Soc. 114 (1965), 176-186. MR 30 \#4920.

11. M. O. Reade, The coefficients of close-to-convex functions, Duke Math. J. 23 (1955), 459-462. MR 17, 1194.

12. T. Sheil-Small, Starlike univalent functions, Proc. London Math. Soc. (3) 21 (1970), 577-613. MR 43 \#2207.

Department of Mathematics, Western Michigan University, Kalamazoo, MiCHIGAN 49001

Department of Mathematics, State University of New York, Brock port, NeW YoRK 14420 